\title{
ON SUBSPACES OF SEPARABLE NORM IDEALS
}

\author{
BY J. R. HOLUB ${ }^{1}$ \\ Communicated by Robert G. Bartle, August 17, 1972
}

1. Let $\mathscr{H}$ be a separable infinite-dimensional Hilbert space and let $\mathscr{L}(\mathscr{H})$ denote the space of all bounded linear operators on $\mathscr{H}$. In the study of operators on Hilbert space and its many applications, particularly in mathematical physics, certain ideals of the ring $\mathscr{L}(\mathscr{H})$ are of fundamental importance. These are the ones called norm ideals by Schatten [11] and s.n. ideals (symmetric norm ideals) by Gohberg and Krein [5]. Examples of such ideals are the space $K(\mathscr{H})$ of compact operators, the space $N(\mathscr{H})$ of nuclear (or trace-class) operators, the Hilbert-Schmidt operators and their natural extensions, the classes $C_{p}(1 \leqq p<+\infty)[3]$, [9], and the rather recently introduced classes $C_{\Omega}^{0}$ and $C_{\omega}$ of Gohberg and Krein [5], [8]. The last are already of considerable importance, arising naturally in the study of the abstract triangular integral and in questions concerning the completeness of the root vectors of an operator [1], [6], [7], [8], yet have barely been studied in any depth. In fact, though all of the ideals mentioned above are separable Banach spaces, almost no work concerning their subspace structure has been done (one exception is the fine study of the ideals $C_{p}$ by McCarthy [9]).

The purpose of this paper is to begin a study of the subspaces of separable norm ideals (by subspace we always mean a closed linear submanifold). In particular, we study the spaces $K(\mathscr{H}), N(\mathscr{H}), C_{\Omega}^{0}$, and $C_{\omega}$ from this standpoint. We refer the reader to [5] for the necessary background information and notation concerning s.n. ideals. Details of the proofs will appear elsewhere.

2. The ideals $K(\mathscr{H})$ and $C_{\Omega}^{0}$. Though the ideals $K(\mathscr{H})$ and $C_{\Omega}^{0}$ are dissimilar in many respects, their subspace structures are very much alike.

THEOREM 1. Let $E$ be a closed subspace of $K(\mathscr{H})$. Then either $E$ is isomorphic to $\mathscr{H}$ or it contains a subspace isomorphic to $c_{0}$. If $E$ is isomorphic to $\mathscr{H}$, then it is complemented in $\mathscr{L}(\mathscr{H})$.

The idea of the proof of Theorem 1 is as follows: $K(\mathscr{H})=\mathscr{H}^{*} \otimes_{\alpha} \mathscr{H}$ for a uniform crossnorm $\alpha$ (in fact $\alpha=\lambda$, the "least" crossnorm [11]). Moreover, if $\left(\phi_{i}\right)$ is an orthonormal basis for $\mathscr{H}$, then $\left(\phi_{i} \otimes \phi_{j}\right)$ is a basis

AMS (MOS) subject classifications (1970). Primary 46L15, 46B05, 46B10, 46C10.

Key words and phrases. Operators on Hilbert space, symmetric norm ideal, tensor product of Hilbert spaces.

${ }^{1}$ Research partially supported by NSF grant GP-33778. 
for $\mathscr{H}^{*} \otimes_{\alpha} \mathscr{H}$ [4]. If $E$ is nonreflexive then it cannot be isomorphically embedded in the finite direct sum of Hilbert spaces. Consequently there exists a sequence $\left(T_{i}\right)$ in $E$ with $\left\|T_{i}\right\|=1$ for all $i$ such that $i:\left\|T_{i} \phi_{n}\right\|+\left\|T_{i}^{*} \phi_{n}\right\|$ $\rightarrow 0$ for each $n=1,2, \ldots$. Using this fact, stability theorems from basis theory [12], and the definition of the norm in $K(\mathscr{H})$, one can show that a subsequence of $\left(T_{i}\right)$ is a basic sequence similar to the unit vector basis of $c_{0}$, implying $c_{0} \subset E$.

If $E$ is reflexive then no such sequence $\left(T_{i}\right)$ can exist in $E$ and so $E$ embeds in a finite direct sum of Hilbert spaces and hence is itself isomorphic to a Hilbert space. The embedding extends to a continuous mapping of $\mathscr{L}(\mathscr{H})$ into the direct sum and from this one obtains the desired projection of $\mathscr{L}(\mathscr{H})$ onto $E$.

Having proved Theorem 1, we can prove that a similar situation holds for $C_{\Omega}^{0}$.

THEOREM 2. Let $E$ be a closed subspace of $C_{\Omega}^{0}$. Then either $E$ is isomorphic to $\mathscr{H}$ or $E$ contains $c_{0}$. If $E$ is isomorphic to $\mathscr{H}$ then $E$ is closed in $\mathscr{L}(\mathscr{H})$ and moreover is complemented there. In particular $E$ is complemented in $C_{\Omega}^{0}$.

To prove Theorem 2 we consider the injection $i: E \rightarrow K(\mathscr{H})$. If $i$ is an isomorphism then the theorem follows from Theorem 1 . If $i$ is not an isomorphism, then there is a sequence $\left(T_{i}\right)$ in $E$ with $\left\|T_{i}\right\|_{\Omega}=1$ for all $i$ but for which $\left\|T_{i}\right\| \rightarrow 0$. Using this fact, the stability theorem, and a rather subtle argument based on the properties of the norm on $C_{\Omega}^{0}$, one can show that a subsequence of $\left(T_{i}\right)$ is again basic and similar to the unit vector basis of $c_{0}$. If $E$ is isomorphic to $\mathscr{H}$, then we must have $E$ closed in $K(\mathscr{H})$ and the second part of the theorem follows from Theorem 1.

3. The ideals $N(\mathscr{H})$ and $C_{\omega}$. Since $N(\mathscr{H})=K(\mathscr{H})^{*}[11]$ and $C_{\omega}=\left(C_{\Omega}^{0}\right)^{*}$ [5], the results of this section are not entirely surprising in view of Theorems 1 and 2. However just as, in general, a knowledge of the subspaces of a Banach space $X$ tells one little about the subspaces of $X^{*}$, so too here we cannot appeal to Theorems 1 and 2 to give the results directly.

THEOREM 3. Let $E$ be a closed subspace of $N(\mathscr{H})$. Then $E$ is either isomorphic to $\mathscr{H}$ or $E$ contains a subspace isomorphic to $l^{1}$. Moreover if $E$ is isomorphic to $\mathscr{H}$ then $E$ is closed in $\mathscr{L}(\mathscr{H})$ and complemented there.

To prove Theorem 3 we consider the injection $i: E \rightarrow H S(\mathscr{H})$ (the Hilbert-Schmidt operators on $\mathscr{H}$ ). If $E$ is not isomorphic to $\mathscr{H}$ then $i$ is not an isomorphism so there exists a sequence $\left(T_{i}\right)$ in $E$ with $\left\|T_{i}\right\|_{N}=1$ for all $i$ but for which $\left\|T_{i}\right\|_{H S} \rightarrow 0$. Now essentially the same argument as was used in Theorem 1 (the only difference being that the nuclear norm determines that the subsequence be similar to $\left(e_{i}\right)$ in $l^{1}$ rather than in $\left.c_{0}\right)$ establishes the first part of the theorem. 
If $E$ is isomorphic to $\mathscr{H}$ then the injection of $E$ into $K(\mathscr{H})$ must also be an isomorphism and an application of Theorem 1 proves the second part of the theorem.

Again, a similar result holds for the ideals $C_{\omega}$.

THEOREM 4. Let $E$ be a closed subspace of $C_{\omega}$. Then either $E$ is isomorphic to $\mathscr{H}$ or E contains a subspace isomorphic to $l^{1}$.

The bulk of the proof is similar to that of the previous theorems. However here we need to also prove the following result (which, incidentally, has as a trivial corollary the result in [10]):

Proposition 5. Let $\lambda_{\omega}$ be the sequence space associated with $C_{\omega}$ (i.e., $\left.\left(a_{i}\right) \in \lambda_{\omega} \Leftrightarrow \sum a_{i} \phi_{i} \otimes \psi_{i} \in C_{\omega}\right)$. Then any closed infinite-dimensional subspace of $\lambda_{\omega}$ contains $l^{1}$.

Finally, we remark that the methods employed in this paper are by no means limited to the investigation of spaces of operators on a Hilbert space. For example, these methods also yield the following.

THEOREM 6. If $E$ is a nonreflexive subspace of $K\left(L^{p}[0,1], L^{s}[0,1]\right)$ $(1<p \leqq 2,2 \leqq s<+\infty)$ then $E$ contains $c_{0}$.

\section{REFERENCES}

1. M. S. Brodskiı̆, I. C. Gohberg, M. G. Kreĭn and V. I. Macaev, Some investigations in the theory of non-selfadjoint operators, Proc. Fourth All-Union Math. Congress, vol. II: Sectional Lectures, "Nauka", Leningrad, 1964, pp. 261-271; English transl., Amer. Math. Soc. Transl. (2) 65 (1967), 237-251. MR 36 \# 3153.

2. J. W. Calkin, Two-sided ideals and congruences in the ring of bounded operators in Hilbert space, Ann. of Math. (2) 42 (1941), 839-873. MR 3, 208.

3. N. Dunford and J. T. Schwartz, Linear operators. II: Spectral theory. Selfadjoint operators in Hilbert space, Interscience, New York, 1963. MR 32 \# 6181.

4. J. Gil de Lamadrid, Uniform cross norms and tensor products of Banach algebras, Duke Math. J. 32 (1965), 359-368. MR 32 \# 8125.

5. I. C. Gohberg and M. G. Krein, Introduction to the theory of linear nonselfadjoint operators in Hilbert space, "Nauka", Moscow, 1965; English transl., Transl. Math. Monographs, vol. 18, Amer. Math. Soc., Providence, R.I., 1969. MR 36 \# 3137; MR 39 \# 7447.

6. - On the theory of triangular representations of nonselfadjoint operators, Dokl.

Akad. Nauk SSSR 137 (1961), 1034-1037 = Soviet Math. Dokl. 2 (1961), 392-396. MR 25 \#3370.

7. - Volterra operators whose imaginary component belongs to a given class, Dokl. Akad. Nauk SSSR 139 (1961), 779-782=Soviet Math. Dokl. 2 (1961), 983-985. MR 25 \#3371.

8. V. I. Macaev, On a class of completely continuous operators, Dokl. Akad Mauk SSSR 139 (1961), 548-551 = Soviet Math. Dokl. 2 (1961), 972-975. MR 24 \# A1617.

9. C. A. McCarthy, Cp., Israel J. Math. 5 (1967), 249-271. MR 37 \# 735.

10. J. R. Retherford, A semishrinking basis which is not shrinking, Proc. Amer. Math. Soc. 19 (1968), 776. MR 37 \#739.

11. R. Schatten, Norm ideals of completely continuous operators, Ergebnisse der Mathematik und ihrer Grenzgebiete N.F., Heft 27, Springer-Verlag, Berlin, 1960. MR 22 \#9878.

12. I. Singer, Bases in Banach spaces. I, Springer-Verlag, New York and Berlin, 1970.

Department of Mathematics, Virginia Polytechnic Institute And State UniverSITY, BLACKSBURG, VIRGINIA 24061 